\title{
Téoros
}

Revue de recherche en tourisme

\section{Le développement du tourisme dans le département de Sololá au Guatemala}

\section{Louis Jolin et Juliana Chaves Dos Santos}

Volume 29, numéro 2, 2010

URI : https://id.erudit.org/iderudit/1024882ar

DOI : https://doi.org/10.7202/1024882ar

Aller au sommaire du numéro

Éditeur(s)

Université du Québec à Montréal

ISSN

0712-8657 (imprimé)

1923-2705 (numérique)

Découvrir la revue

Citer cette note

Jolin, L. \& Chaves Dos Santos, J. (2010). Le développement du tourisme dans le département de Sololá au Guatemala. Téoros, 29(2), 151-155.

https://doi.org/10.7202/1024882ar d'utilisation que vous pouvez consulter en ligne. 


\title{
Le développement du tourisme dans le département de Sololá au Guatemala
}

\author{
Louis JOLIN \\ Professeur, département d'études urbaines et touristiques \\ École des sciences de la gestion, Université du Québec à Montréal \\ jolin.louis@uqam.ca \\ Juliana CHAVES DOS SANTOS \\ Maîtrise en développement du tourisme \\ École des sciences de la gestion, Université du Québec à Montréal \\ chaves_dos_santos.juliana@courrier.uqam.ca
}

Depuis 2008, le Centre d'étude et de coopération internationale (CECI) et la Société de coopération pour le développement international (SOCODEVI) sont les maîtres d'œuvre d'un projet de coopération dans le département de Sololá au Guatemala, financé par l'Agence canadienne de développement international. Ce projet (PROSOL), a pour objectif "d'améliorer les conditions de vie et d'augmenter le revenu moyen de 30000 familles du département de Sololá, l'une des régions autochtones les plus pauvres du Guatemala» (ACDI, 2010). Plusieurs volets furent retenus : l'agriculture, l'artisanat, l'environnement et le tourisme.

Le Projet de développement économique rural du département de Sololá (PROSOL) est le résultat de plusieurs études commandées par l'ACDI pour la relance économique du Guatemala entre 2001 et 2005. Des diagnostics sectoriels concernant le département de Sololá réalisés par SOCODEVICECI en 2006-2007, durant la phase de conception du projet, ont identifié certains domaines comme leviers du développement du département et comme générateurs d'emplois. Ces domaines sont l'agriculture (principalement au niveau de la production de café et de légumes), le tourisme et le développement de microentreprises (dans l'artisanat et autres secteurs). La phase d'exécution du projet est prévue pour sept ans, de 2008 à 2015.

En février 2009, on a effectué le lancement officiel du PROSOL au Guatemala. De novembre 2008 à la fin de 2009, l'équipe du projet a mis en place des partenariats avec les principaux intervenants de la région. En plus, elle a complété le plan de mise en ouvre et des axes de travail (SOCODEVI, 2010a).

Le Centre international de formation et de recherche en tourisme (CIFORT) de l'École des sciences de la gestion (ESG) de l'Université du Québec à Montréal (UQAM) a été approché pour appuyer l'équipe locale dans la mise en œuvre d'un certain nombre de priorités de développement dans le secteur du tourisme.
Le présent texte est divisé en quatre parties :

- le tourisme au Guatemala et plus particulièrement aux abords du lac Atitlán (département de Sololá);

- les axes prioritaires du PROSOL pour le développement touristique;

- les axes d'une collaboration possible avec le CIFORT;

- un exemple concret de la collaboration.

\section{Le tourisme au Guatemala et aux abords du lac Atitlán}

Le Guatemala, pays d'Amérique centrale, a une population de 13276517 habitants dont 40,6\% sont d'origine uniquement indigène (Maya) et dont $40 \%$ parlent les langues amérindiennes (il y a 23 langues amérindiennes officielles) (CIA, 2009). Le pays est divisé en 22 départements, le Petén étant le plus grand. Après une guerre civile de plus de 35 ans, le Guatemala tente désormais de se reconstruire sur une base démocratique. Il est le pays le plus peuplé d'Amérique centrale et possède un PIB de 5300 \$ US par personne (CIA, 2009).

Le département de Sololá possède une superficie de $12230 \mathrm{~km}^{2}$, équivalente à $11 \%$ du territoire national. Situé entre 1591 à $3537 \mathrm{~m}$ d'altitude, Sololá partage des frontières avec cinq autres départements : Quiché, Totonicapán, Quetzaltenango, Chimaltenango et Suchitepéquez. Le département de Sololá a 19 communes, dont 12 qui sont situées autour de lac Atitlán.

La région autour du lac est considérée comme l'une des destinations touristiques principales du Guatemala (INGUAT et BID, 2007a). Parmi les 12 municipalités autour du lac, Panajachel, Santiago et San Pedro sont les plus visitées.

Le lac Atitlán a comme produit spécifique la culture maya vivante, présente dans les articles d'artisanat, les repas typiques, les langues parlées et surtout dans les vêtements de la population. Le développement touristique dans cette région est soumis à de fortes contraintes. Parmi celles-ci, on peut mentionner le manque de réseaux d'assainissement des eaux, 


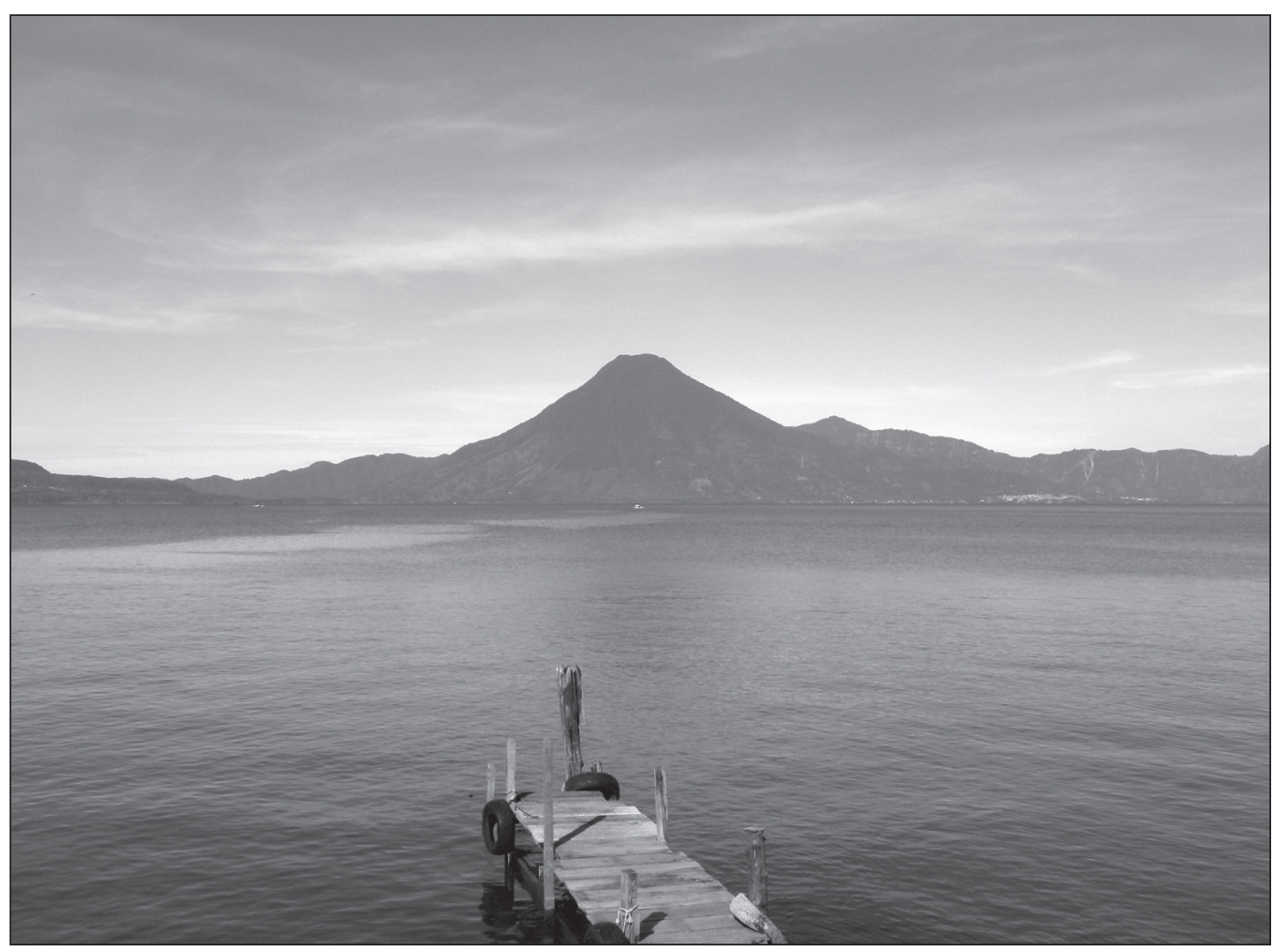

ILLUSTRATION 1 : Vue du Lac Atitlán. Photo prise à Panajachel (photo : Juliana Chaves). une planification territoriale et urbaine désordonnée, l'absence d'un transport public adéquat, un accès déficient à une éducation publique et à un système de santé de qualité pour tous, etc. Plusieurs de ces problèmes affectent non seulement la région du lac, mais tout le Guatemala ainsi que d'autres pays en Amérique latine. Il est très important de tenir compte de la fragilité et des insuffisances de l'environnement dans lequel on souhaite promouvoir et développer le tourisme. Un exemple de cette fragilité a été révélé par le passage de la tempête tropicale Agatha à l'été 2010, qui a provoqué plusieurs éboulements, la destruction de ponts, et qui a déversé des ordures dans les rues de Panajachel (le village qui reçoit la plupart des visiteurs du lac) et le long du lac, sans compter la grande quantité de familles délogées et les décès. Il est aussi important de mentionner le manque de sécurité du pays, ce qui affecte son image face au marché mondial du tourisme.

La région du lac Atitlán offre une large gamme d'activités touristiques reliées à la nature, à la culture et à l'éducation. Une liste des activités a été énoncée dans le Plan Estratégico de dinamización turística para el Lago de Atitlán (INGUAT et BID, 2007a) :

- Activités reliées à la nature : visites guidées, observation et interprétation de la nature, observation d'oiseaux, pratique de la photographie de la nature, randonnées pédestres, escalade, vélo, randonnées équestres, pratique du kayak, plongée sous-marine et baignade;

-Activités culturelles : visites de monuments religieux, visites de musées, centres et producteurs d'artisanat, participation à des rituels mayas et visites de centres d'interprétation de la culture maya;
- Activités d'éducation : les établissements d'enseignement de la langue espagnole représentent un élément d'attrait touristique.

\section{Les axes prioritaires du PROSOL pour le développement touristique}

Pendant la phase conceptuelle, plusieurs rapports ont été rédigés. Dès 2006, SOCODEVI en partenariat avec le CECI a réalisé une étude intitulée Desarrollo del Turismo en Sololá (PROSOL, 2006). En 2007, CASABAL, un groupe de consultants en tourisme du Guatemala, a rédigé un document intitulé Sector económico - Turismo s'inscrivant dans le plan de développement de la région, le "Proyecto desarrollo económico rural de Sololá» (CASABAL, 2007). Ce plan propose une stratégie de développement touristique basée sur six volets : l'amélioration de l'image urbaine, le développement du tourisme communautaire, la promotion de l'investissement touristique, la diversification et l'amélioration de la qualité des produits touristiques, la formation et le développement des ressources humaines, et la valorisation de la culture, de la communication, de la promotion et du marketing.

Presque en même temps, l'Instituto Guatemalteco de Turismo (INGUAT) présentait son plan de développement du tourisme pour le département de Sololá, le Plan Estratégico de dinamización turística para el Lago de Atitlán (INGUAT et BID, 2007b). Ce plan est divisé en six axes et 17 stratégies de développement. Pour chacune des stratégies, il y a une série d'actions identifiées pour la réalisation du projet. Il y a en tout 32 programmes et 63 actions qui ont été identifiés pour la région du lac Atitlán (INGUAT et BID, 2007b). Plusieurs 
ILLUSTRATION 2 : Vente de produits à Santa Catarina Palopo (photo : Juliana Chaves).

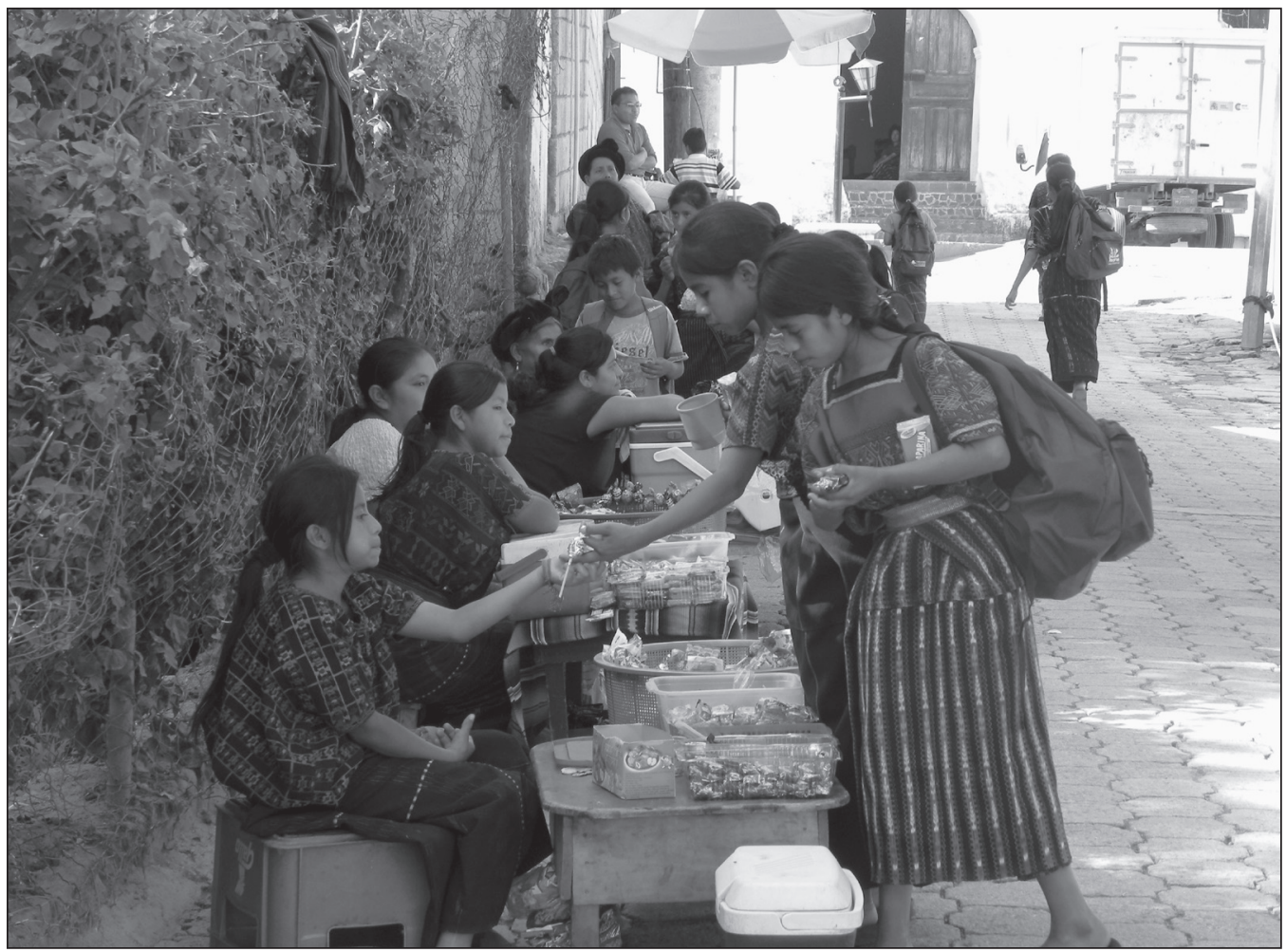

entreprises liées à l'industrie touristique ont contribué à l'élaboration du plan.

Au fil des travaux, les axes prioritaires du PROSOL en tourisme furent précisés et nuancés par l'équipe locale chargée du volet tourisme. Ils peuvent se résumer de la manière suivante :

- un appui aux institutions locales dans le secteur du tourisme afin d'améliorer la gouvernance dans ce secteur (ex. : un appui à la Commission de tourisme de Sololá);

- l'amélioration de l'image urbaine (ex. : la Calzada Venancio Barrios à Sololá);

- le développement du tourisme communautaire;

- l'accroissement des investissements dans le secteur du tourisme;

- la diversification et l'amélioration de la qualité des produits touristiques (ex. : le parc municipal Chuiraxamolo);

- la formation et le développement des ressources humaines (par l'Universidad del Valle et INTECAP);

- le développement de l'entreprenariat;

- l'amélioration de la communication, de la promotion et du marketing touristiques (amélioration du site internet, carte touristique, etc.).

\section{Les axes d'une collaboration possible avec le CIFORT}

Le CIFORT peut apporter son concours pour la réalisation d'un certain nombre d'objectifs de PROSOL dans le domaine du tourisme. Voici quelques exemples :

1) La formation des ressources humaines et la recherche en tourisme :
- en collaborant au développement du programme de tourisme du campus Altiplano de l'Universidad del Valle, la «carrera técnica universitaria en turismo»;

- en s'associant à diverses recherches nécessaires en partenariat avec le Centre de recherches de l'Universidad del Valle, principalement en ce qui concerne le tourisme durable et l'écotourisme;

-en participant concrètement par ses professeurs et chargés de cours à des formations de formateurs;

- en intéressant des étudiants du baccalauréat en gestion du tourisme et de l'hôtellerie, et de la maîtrise en développement du tourisme de l'ESG UQAM à réaliser des stages au Guatemala selon les besoins identifiés par le PROSOL et ses partenaires, principalement pour tout ce qui touche le soutien à apporter au tourisme communautaire.

2) Le partenariat avec des organisations spécialisées en tourisme d'envergure internationale :

- en facilitant les contacts des partenaires guatémaltèques et du PROSOL avec des organisations comme le programme ST-EP de l'Organisation mondiale du tourisme (OMT), le Centre d'excellence des destinations (CED), l'Organisation internationale du tourisme social (OITS/BITS)...

3) Divers autres sujets comme l'amélioration de l'image urbaine ou la mise en place d'un réseau d'accueil et d'informations touristiques pour le département :

- en fournissant de l'expertise et en établissant divers contacts auprès d'interlocuteurs québécois qui pourraient apporter leur collaboration.

Pour officialiser cette collaboration, une entente générale entre le CIFORT et SOCODEVI est en voie de conclusion. 
D'ores et déjà depuis 2009, quelques missions furent réalisées : celle du professeur Louis Jolin pour identifier certaines priorités en termes de formation de ressources humaines et pour préciser les termes de la collaboration avec le CIFORT; celles de madame Marie-Andrée Delisle, consultante et diplômée de la maîtrise en tourisme de l'UQAM, concernant les priorités en termes de développement du tourisme communautaire; et enfin, à l'été 2010, le stage de recherche d'une étudiante en maîtrise, madame Juliana Chaves dos Santos, qui portait sur un inventaire et une évaluation des initiatives de tourisme communautaire.

\section{Un exemple concret de la collaboration}

Le stage de madame Chaves dos Santos a permis de vérifier de manière globale la situation du tourisme communautaire dans les communautés visitées, la perception qu'en ont les agences de voyages de Panajachel, et le travail des ONG et des agences de coopération internationale dans les projets de tourisme communautaire.

Selon Delisle et Jolin (2007), le tourisme communautaire accorde une place importante au processus de décision par les communautés d'accueil elles-mêmes en ce qui concerne les visiteurs à accueillir et les modalités de leur intégration. Ces auteurs (2007 : 51) expliquent que «le tourisme communautaire, qui s'appuie très souvent sur l'engagement des femmes, vise surtout à apporter aux communautés une diversification économique, à favoriser la protection d'un patrimoine humain naturel et encore à revitaliser un lieu, une communauté et son économie par l'activité touristique».

D'autres travaux (Laurent, 2003; RAPAC, 2009) apportent des nuances à cette définition, mais vont dans le même sens pour l'essentiel.

Suite à la mission de Juliana Chaves dos Santos, qui s'est réalisée dans un contexte difficile - le département de Sololá ayant eu à affronter à l'été 2010 des tempêtes tropicales qui ont affecté les infrastructures -, on peut tirer quelques conclusions. Ces conclusions, résultant d'un travail d'observation et d'entrevues semi-dirigées avec les responsables d'initiatives de tourisme communautaire, permettent de mieux cibler les interventions futures pour un meilleur développement du tourisme communautaire dans le département de Sololá. Certaines des conclusions ci-dessous sont appuyées également par d'autres études sur le développement touristique de la région :

- Un manque de coordination a été noté entre les initiatives de chaque communauté et entre les municipalités. Afin d'augmenter le séjour des touristes qui visitent la région du lac Atitlán, les communautés et les initiatives touristiques devraient s'unir pour mener à bien la promotion et la commercialisation de leurs produits et services.

- Une absence d'équilibre a été relevée entre l'offre et la demande - il y a des lieux où l'offre est supérieure à la demande et vice versa.

- Les responsables des initiatives de tourisme communautaire ont peu de connaissance des techniques de promotion et de commercialisation de leurs produits.

- Les associations de jeunes guides ne sont pas suffisamment préparées pour recevoir les touristes : on constate un manque de connaissance de la langue anglaise.
- Étant donné le faible niveau de développement de la majorité des initiatives touristiques de la région, une des solutions pour atteindre un meilleur positionnement dans le marché interne serait de viser le marché du tourisme solidaire ou équitable.

- Les groupes et les organisations qui travaillent pour le développement du tourisme devraient être plus interconnectés afin d'unir leurs efforts dans le but d'augmenter l'efficacité de leurs actions ainsi que les bénéfices pour les communautés.

- Une insuffisance d'une vision commerciale du tourisme dans les décisions et les actions de la Commission du tourisme du département de Sololá a été remarquée. Une explication de cet état de fait vient peut-être de la présence de plusieurs membres faisant partie d'ONG au sein de l'organisation. Les initiatives de tourisme communautaire ont besoin d'améliorer leur vision commerciale. Le tourisme, c'est une affaire qui doit être traitée de cette manière même s'il doit rester sous le contrôle de la communauté avec des préoccupations de développement durable.

Les conclusions ont été adressées à la Commission du tourisme de Sololá (COMTUR) qui a accueilli la stagiaire. Cette commission fut créée dans le but de décentraliser les actions, mais elle reste encore trop peu connue de plusieurs acteurs locaux, ce qui ne permet pas toujours la mise en ouvre d'actions effectives et concrètes. Par exemple, la planification des stratégies de promotion et de commercialisation de la destination Atitlán élaborées par les membres de la COMTUR ne prend pas toujours en compte les stratégies développées par les municipalités.

Ce travail réalisé par une stagiaire du programme de mâ̂trise en développement du tourisme de l'ESG UQAM est un exemple de la collaboration entre le CIFORT et l'équipe locale du PROSOL. D'autres actions sont à venir.

\section{Références}

ACDI (2009) «À propos de l'ACDI», Ottawa : Agence canadienne de développement international, $<$ http://www.acdi-cida.gc.ca/acdi-cida/ACDICIDA.nsf/fra/NIC-5313423-N2A>, consulté le 12 janvier 2009.

ACDI (2010) «Développement du secteur privé dans le département de Sololá», Ottawa : Agence canadienne de développement international, <http://www.acdi-cida.gc.ca/cidaweb/cpo.nsf/vLUWebProjFr/1A 95BD13FFA817E38525749800371306?OpenDocument $>$, consulté le 9 novembre 2010.

CASABAL (2007) Sector económico — Turismo, étude réalisé pour le Projet de développement économique rural de Sololá (PROSOL), Guatemala City : CASABAL. 23 p.

CECI (s. d.) «Nos projet par région : Guatemala — Création d'emplois et accroissement des revenus», Montréal : Centre d'étude et de coopération internationale, $<$ http://www.ceci.ca/fr/ra2010/developpement-local/guatemala-creation-d-emplois-et-accroissement-des-revenus/>, consulté le 2 février 2010.

CIA (2009) «Library: Publication: The world Factbook: Guatemala», États-Unis : Central of Intelligency of America, $<$ https://www.cia.gov/ library/publications/the-world-factbook/geos/gt.html>, consulté le 21 octobre 2009. 
DELISLE, Marie-Andrée (2009) Le tourisme communautaire au Lac Atitlán, Stratégies de développement, rapport de l'étude sollicité par PROSOL, Montréal : Marie-Andrée Delisle, octobre, document non publié.

DELISLE, Marie-Andrée et Louis JOLIN (2007) Un autre tourisme est-il possible?, Québec : Presses de l'Université du Québec. 144 p.

GSTC - Global Sustenable Tourism Criteria (2010) Criteria Background \& History, TSC, <http://www.sustainabletourismcriteria.org/index. php?option=com_content\&task=view\&id=164\&Itemid=348>, consulté le 5 juin 2010.

INGUAT et BID (2007a) Diagnostico - Plan Estratégico de dinamización turística para el Lago Atitlán, Guatemala City : Instituto Guatemalteco de Turismo, étude réalisée par le Grupo GDT. 149 p.

INGUAT et BID (2007b) Plan Estratégico de dinamización turística para el Lago Atitlán, Guatemala Ciy : Instituto Guatemalteco de Turismo, étude réalisée par le Grupo GDT. 116 p.

JOLIN, Louis et Monique BOUCHARD (2008) Résumés des plans de développement du lac Atitlán, Montréal : UQAM, document non publié. 43 p.

LAURENT, Alain (2003) «Caractériser le tourisme responsable facteur de développement durable», France : BEIRA.CFP groupe T2D2 Tourismes, Territoires et Développement Durable. 270 p. $<\mathrm{http}: / / \mathrm{www}$. tourisme-solidaire.org/ressource/etude_laurent.htm $>$, consulté le 10 décembre 2009.
PROSOL — Projet de développement économique rural de Sololá (2006) Desarrollo del Turismo en Sololá, Guatemala : Consortium SOCODEVICECI. 105 p.

Rainforest Alliance (2010) Tourism, Sustanable Tourism, New York : Rainforest Alliance, $<$ http://www.rainforest-alliance.org/tourism. cfm?id=main $>$, consulté le 5 mai 2010.

RAPAC, Réseau des aires protégées d'Afrique centrale (2009) «Bibliothèque, outils pratiques, développement touristique, Écotourisme et Tourisme Communautaire pour les Aires Protégées d'Afrique centrale : Manuel», RAPAC. 108 p. <http://www.rapac.org/index.php/biblio/cat_view/87outils-pratiques/89-developpement-touristique>, consulté le 11 novembre 2009.

SOCODEVI (2010a) «Nos Projet: Projet de développement économique rural du département de Sololá (PROSOL)», Québec : Société de coopération pour le développement international, $<\mathrm{http}$ ://www.socodevi.org/ fr/projets/_detail_projet.php?pk_projet=108\&secteurAct=8>, consulté le 12 janvier 2010.

SOCODEVI (2010b) «Communiqué - Diminution de la pauvreté et Création d'emploi au Guatemala", Québec : Société de coopération pour le développement international, $<$ http://www.socodevi.org/_userfiles/file/Communiques/20090225_COM_SololaCECI-SOCODEVI. pdf $>$, consulté le 12 janvier 2010.

\section{Appel à textes}

En dehors des dossiers thématiques, Téoros ouvre ses pages aux propositions spontanées. La rédaction invite tous les chercheurs qui œuvrent dans le domaine du tourisme ou qui s'intéressent au tourisme sous tous ses aspects à soumettre des articles de nature analytique à la revue. On peut soumettre un article en l'envoyant à :

\section{teoros@uqam.ca}

Les textes soumis doivent apporter une contribution scientifique originale, que ce soit par le biais d'information factuelle jusqu'alors inconnue ou par une nouvelle interprétation d'un thème particulier. Téoros vise avant tout le transfert de connaissances; son objectif est donc de promouvoir une meilleure compréhension des phénomènes liés au tourisme.

Les auteurs doivent faire parvenir un manuscrit présenté selon les règles de la revue, disponibles au www.teoros.uqam.ca. Un article analytique compte environ 6000 mots et n'excède pas 8000 mots, avec trois ou quatre illustrations en 300 ppp.
Les articles peuvent être soumis en anglais ou en français et doivent être accompagnés d'un résumé de 200 mots et de cinq mots-clés.

La publication des articles se fait sous réserve d'une évaluation. Tous les manuscrits seront évalués anonymement par des pairs, qui pourront faire des suggestions ou demander des modifications. La rédaction transmettra l'avis des évaluateurs aux auteurs et s'assurera que les modifications demandées seront apportées.

Au plaisir de vous lire dans nos pages.

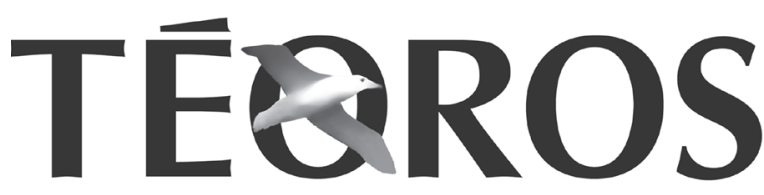

REVUE DE RECHERCHE EN TOURISME 